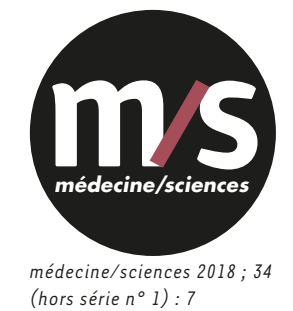

\title{
Les rencontres RARE 2017 vues du point de vue des industriels de la santé
}

Antoine Ferry

> Madame la ministre de la Santé et des Solidarités a montré l'intérêt qu'elle porte aux maladies rares en inaugurant les rencontres RARE. Elle peut également compter sur les industriels du médicament pour faire face aux nombreux défis qui nous attendent aux côtés de la communauté des maladies rares. Cette communauté a toujours fonctionné de manière exemplaire, a permis de co-construire positivement et de remporter de premiers défis importants. Je peux vous assurer que les industriels soutiendront en permanence la recherche et les innovations. Je remercie les équipes de la Fondation Maladies rares et en particulier Audrey Tranchand, qui ne compte pas ses heures depuis de nombreux mois. Grâce à elle, nous avons pu relever ce défi en moins d'un an, nous permettant d'être aujourd'hui réunis à la Cité des Sciences, lieu hautement symbolique, notamment par sa dénomination. Je remercie également les membres du Conseil scientifique pour leur contribution et pour leur disponibilité. Enfin, je tenais à rendre hommage à Gilles Roche et à Eurobiomed, qui ont porté les premières rencontres Rare sur les fonts baptismaux il y a huit ans.

La France a toujours été leader dans le domaine des maladies rares, que ce soit dans l'organisation des soins et l'excellence de la recherche ou dans la mise à disposition des produits, dans le seul intérêt des patients. L'innovation thérapeutique en France et en Europe s'accélère considérablement depuis les années 2010, avec de nombreuses innovations de rupture, principalement dans les maladies rares et en oncologie. Pour autant, les besoins de santé publique non couverts restent encore immenses. Au regard du nombre de médicaments disponibles et du nombre de maladies rares, cette dynamique d'innovation a besoin d'être soutenue par un environnement économique attractif en France. Nous pouvons et nous devons aller encore beaucoup plus loin que les 150 médicaments existant aujourd'hui. Ce soutien est attendu aussi bien des grandes entreprises que par les petites structures innovantes de plus en plus impliquées dans les maladies rares. Dans un contexte économique de plus en plus contraint, l'excellence de ce modèle ne doit pas être mise à mal. La logique comptable a ses raisons, mais elles ne doivent pas déstructurer cette belle dynamique. Nous devons tous trouver des solutions concrètes. J'espère que ces deux jours permettront de définir de grandes priorités qui viendront enrichir le futur plan Maladies rares.

L'évaluation des médicaments destinés à traiter les maladies rares présente de très nombreuses spécificités qui rendent les procédures d'évaluation en vigueur très souvent inadaptées. Ce sera encore plus vrai demain avec des thérapies en rupture totale avec l'existant, comme les thérapies

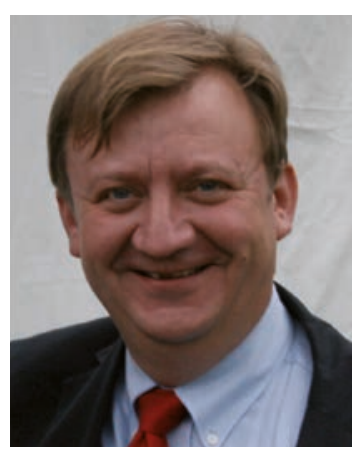

Vice-président du Comité Maladies Rares du LEEM, Laboratoires CTRS, 63 , rue de l'Est 92100 Boulogne Billancourt, France.

aferry@ctrs.fr

cellulaires et les thérapies géniques. II s’ensuit des négociations économiques interminables, voire des situations d'impasse, et les prix proposés sont hélas très souvent déconnectés des prix européens. Enfin, les Autorisations Temporaires d'Utilisation (ATU), enviées par nos voisins européens, sont de plus en plus exposées du fait de contraintes de plus en plus importantes, risquant de limiter leur nombre dans les années à venir. Nous devons donc tous nous mettre autour de la table pour éviter de plonger dans une spirale qui reléguerait la France dans une position peu enviable. II ne faut jamais oublier que les maladies rares s'affirment aujourd'hui comme l'un des plus grands axes de l'innovation de demain, pour les maladies rares mais aussi pour d'autres maladies beaucoup plus fréquentes, qui bénéficient souvent de l'amélioration des connaissances sur les mécanismes physiopathologiques.

Si notre attractivité diminue, nous perdrons toutes les avancées conquises depuis le premier Plan : le recrutement dans les études cliniques diminuera, la recherche sur le territoire national ne sera plus une priorité et les ATU seront délaissées. Si telle est la volonté politique, il faut l'annoncer clairement. On peut être rassuré par le discours ministériel et faire confiance à ce nouveau gouvernement qui a mis l'innovation et la santé au cœur de ses priorités, pour mettre en place des actions concrètes, efficaces et rapides. $\diamond$

The RARE 2017 meeting for companies developing products for rare patients

\section{LIENS D'INTÉRÊT}

L'auteur déclare n'avoir aucun lien d'intérêt concernant les données publiées dans cet article. 